\title{
GATA6 suppression enhances lung specification from human pluripotent stem cells
}

\author{
Chia-Min Liao, ${ }^{1,2}$ Somdutta Mukherjee, ${ }^{2}$ Amita Tiyaboonchai, ${ }^{2}$ Jean Ann Maguire, ${ }^{1,2}$ Fabian L. Cardenas-Diaz, ${ }^{1,2}$ \\ Deborah L. French, ${ }^{1,2}$ and Paul Gadue ${ }^{1,2}$ \\ 'Department of Pathology and Laboratory Medicine, and 'Center for Cellular and Molecular Therapeutics, The Children's Hospital of Philadelphia, Philadelphia, Pennsylvania, USA.
}

\begin{abstract}
The transcription factor CATA6 has been shown to be important for lung development and branching morphogenesis in mouse models, but its role in human lung development is largely unknown. Here, we studied the role of CATA6 during lung differentiation using human pluripotent stem cells. We found that the human stem cell lines most efficient at generating NKX2.1+ lung progenitors express lower endogenous levels of GATA6 during endoderm patterning and that knockdown of CATA6 during endoderm patterning increased the generation of these cells. Complete ablation of CATA6 resulted in the generation of lung progenitors displaying increased cell proliferation with up to a 15-fold expansion compared with control cells, whereas the null cell line displayed a defect in further development into mature lung cell types. Furthermore, transgenic expression of CATA6 at the endoderm anteriorization stage skewed development toward a liver fate at the expense of lung progenitors. Our results suggest a critical dosage effect of GATA6 during human endoderm patterning and a later requirement during terminal lung differentiation. These studies offer an approach of modulating CATA6 expression to enhance the production of lung progenitors from human stem cell sources.
\end{abstract}

\section{Introduction}

Embryonic lung development can be divided into 4 stages: embryonic, pseudoglandular, canalicular, and saccular (1). Several transcription factors have been implicated during these stages in the early embryo, including the master regulator thyroid transcription factor 1 (also known as NKX2.1). Lack of NKX2.1 results in impaired respiratory distal structure and surfactant protein expression (2). Other transcription factors and signaling pathways that have been implicated in controlling respiratory cell fate specification in the early embryo include bone morphogenetic protein (BMP), hedgehog (HH), WNT, and retinoic acid (RA) (3). The level and timing of each factor governs patterning, morphogenesis, and cell fate decisions.

GATA6 is a member of a transcription factor family that can be subdivided into 2 groups. GATA1, GATA2, and GATA3 are involved in hematopoietic cell development (4), and GATA4, GATA5, and GATA6 are mainly expressed and involved in endoderm-derived tissues, as well as in the cardiogenic mesoderm (5). In the mouse lung, GATA6 has been shown to transactivate the NKX2.1 promoter directly, and act as a regulator for several genes expressed selectively in lung development (6). A homozygous GATA6-null mutation leads to lethality in mice, due to a defect in the extra-embryonic endoderm (7). However, GATA6null embryonic stem cells (ESCs) can still contribute to the endoderm in chimeras (8). Studies revealed that GATA6 is required for lung maturation and that inhibition of GATA6 blocks terminal differentiation (9). Lower GATA6 levels seem to be essential for proper lung maturation, as overexpression of GATA6 in post-

Conflict of interest: The authors have declared that no conflict of interest exists. Submitted: July 26, 2017; Accepted: April 17, 2018.

Reference information: / Clin Invest. 2018;128(7):2944-2950.

https://doi.org/10.1172/JCI96539. natal respiratory epithelial cells leads to inhibition of alveolarization and impairs lung function (10). These studies reveal that precise timing and levels of GATA6 are critical for proper lung development and function.

To study the role of GATA6 in human lung development, we used human pluripotent stem cells (PSCs). These cells provide a powerful model to study lung development and generate human lung disease models. Line-to-line variability is an issue with all PSC differentiation protocols, including lung, and the mechanisms regulating early lung lineage commitment are not completely understood. Here, we report that decreased endogenous expression of GATA6 correlates with efficient human lung progenitor generation in vitro. CRISPR/Cas9-mediated GATA6 knockout resulted in the generation of increased lung progenitors (>15-fold), but this line failed to express terminal lung maturation markers. Transient siRNA-mediated GATA6 knockdown resulted in increased lung generation while maintaining normal terminal lung differentiation. Our studies illustrate the importance of GATA6 in human endoderm patterning and early lung development, highlighting an approach for enhancing the production of lung progenitors from human PSCs.

\section{Results and Discussion}

Efficient NKX2.1 lung progenitor generation from human PSCs expressing low levels of GATA6. The different genetic backgrounds of human PSC lines are known to affect the efficiency of differentiation into a wide variety of cell types. This is especially the case for lung progenitor formation, with multiple labs favoring RUES2 from Rockefeller University Embryonic Stem Cell Lines (11). To uncover modulators of lung specification that may be driving these differences, 3 PSC lines were analyzed that we found generate lung progenitors with differing efficiencies. 
Using a modified version of a previously published lung differentiation protocol (Figure 1A) (11), we quantified the generation of lung progenitors by expression of the master regulator of lung development, NKX2.1. Definitive endoderm (DE) cells were purified and specified into lung precursors that were examined at day 13 of differentiation for expression of NKX2.1 (Figure 1B and Supplemental Figure 1, C and D; supplemental material available online with this article; https://doi.org/10.1172/JCI96539DS1). Robust generation of NKX2.1 $1^{+}$progenitors was observed using a WT induced pluripotent stem cell (iPSC) line, iPS1, that we previously described (12). Progenitor generation increased compared with the RUES2 ESC line as well as the MEL1 ESC line (Figure 1B and Supplemental Figure 1, C and D). The iPSC line was originally generated from a patient with pancreas agenesis due to a heterozygous mutation in GATA6, which was corrected by genome editing (12). Considering that pancreas agenesis is only about $60 \%$ penetrant (13), we hypothesized that the patient's genetic background led to lower baseline GATA6 expression, contributing to disease susceptibility. For example, variants in the GATA6 promoter or enhancer could possibly lead to such differences, which could also impact lung development. We found that GATA6 expression at the anteriorization stage of differentiation was lowest in the iPS1 line, highest in the MEL1 line, and intermediate in the RUES2 line (Figure 1C and Supplemental Figure 1, A and B), displaying an anticorrelation with lung progenitor specification. In order to confirm this anticorrelation in a larger cohort of cell lines, we analyzed the differentiation of 3 additional WT cell lines (1 ES and 2 iPS lines), analyzing GATA6 on day 7 versus NKX2.1 expression on differentiation day 13 (Figure 1D). Despite explaining only a moderate fraction of the total variation $\left(r^{2}=0.27\right)$, the anticorrelation is statistically significant $(P=0.01)$, suggesting that low GATA6 levels at the anteriorization stage are related to more robust NKX2.1 upregulation. Examination of GATA4 expression showed a similar pattern to GATA6, whereas FOXA2 expression levels showed a reverse pattern (Supplemental Figure 1B).

To confirm that decreased expression of GATA 6 during anteriorization enhanced the generation of NKX2.1 ${ }^{+}$progenitors, GATA6 expression was decreased using siRNA. Two different siRNAs decreased GATA6 protein levels in greater than $50 \%$ of cells with an average mean fluorescence intensity (MFI) decrease of about $50 \%$ (Figure 1, E and F). On day 13, we observed a significant increase in the generation of NKX2.1 cells, from $5 \%$ to $15 \%-20 \%$ in the GATA6 siRNA-treated groups (Figure 1, G and H). On average, the percentage of NKX2.1+ progenitors increased 3-fold, and the absolute yield of NKX2.1 ${ }^{+}$cells increased 4 -fold in the GATA6 siRNA-treated groups (Figure 1H). Similar results were seen in 2 additional cell lines (Supplemental Figure 2, A-C and Supplemental Figure 3, A-C). These data suggest that lower GATA6 expression enhances the generation of NKX2.1+ progenitors.

To define the stages of lung progenitor generation that were impacted by GATA6 knockdown, $\mathrm{SOX}^{+}$anterior endoderm cells and NKX2.1+ lung-specified cells were analyzed on day 13 . The anterior endoderm expresses SOX2 in the absence of NKX2.1 (SOX $2^{+} \mathrm{NKX} 2.1^{-}$), and NKX2.1 expression is maintained in the proximal lung during the lung progenitor stage $\left(\mathrm{SOX} 2^{+} \mathrm{NKX} 2.1^{+}\right)$, whereas SOX2 expression is lost in the distal lung (SOX2-NKX2.1 $1^{+}$(14). On day 13 , cells treated with the GATA6 siRNAs had significantly increased SOX2 ${ }^{+} \mathrm{NKX} 2.1^{+}$and SOX2-NKX2.1 $1^{+}$progenitor populations, but not anterior endoderm cells (SOX2 $\left.{ }^{+} \mathrm{NKX} 2.1^{-}\right)$(Figure 1, I and J). In cells treated with siRNAs, the absolute yield of NKX2.1 ${ }^{+}$ cells was increased 4- to 5-fold (Figure $1 \mathrm{H}$ ) and costaining for the proliferation marker Ki67 was also increased in the NKX2.1 $1^{+}$population (Figure 1K). Similar results were observed in the iPS1 and RUES2 cell lines (Supplemental Figure 2, D-F and Supplemental Figure 3, D-F). These data suggest that decreased expression of GATA6 during anteriorization and lung patterning leads to increased numbers of lung progenitors, possibly due to proliferation.

Increased NKX2.1+ progenitor generation in GATA6-null human PSCs. To determine if complete loss of GATA6 also impacted lung progenitor formation, a GATA6-null MEL1 ESC line (MEL1 ${ }^{-/}$) was generated using CRISPR/Cas9 (Supplemental Figure 4, A-C and E). GATA6 protein expression was absent in the $\mathrm{MEL1}^{-/-}$line at the DE stage (Figure 2A and Supplemental Figure 4B). Confirming our previous findings, this line demonstrated a mild endoderm defect, generating about $40 \%-70 \% \mathrm{DE}$ (data not shown and ref. 12). The $\mathrm{MEL1}^{-/-}$line yielded a 5- to 20-fold increase in cell yield over the control from days 11-15 (Figure 2B). In addition, SOX2 and NKX2.1 expression were higher over both anteriorization and lung specification in the $\mathrm{MEL1}^{-/}$cells whereas 2 direct targets of GATA6, HNF1B and GATA4, were repressed in the $\mathrm{MEL1}^{-/-}$line (Figure 2C). In addition to increased expression of NKX2.1 in the $\mathrm{MEL1}^{-/-}$ line compared with control $(40 \% \pm 11 \%$ versus $10 \% \pm 1 \%)$ (Figure 2D), a dramatic increase in absolute yield of NKX2.1 cells (15- to 20 -fold) was also observed (Figure 2E). Also, cell proliferation doubled (Figure 2F), with NKX2.1 $1^{+} \mathrm{Ki}^{+}$and NKX2.1-Ki67 ${ }^{+}$cells both being higher in the MEL1 ${ }^{-/}$line (Figure 2G). Despite the 15-fold expansion, we also examined apoptotic markers in the $\mathrm{MEL}^{+/+}$ and $\mathrm{MEL}^{-/-}$lines. Activated Caspase 3 was substantially increased in the $\mathrm{MEL}^{-/-}$line $(12.3 \% \pm 0.3 \%)$ compared with the $\mathrm{MEL}^{+/+}$line $(1.3 \% \pm 0.1 \%)$ (Supplemental Figure $5 \mathrm{~A})$ and there was a significant increase of Annexin $\mathrm{V}^{+}$cells in the $\mathrm{MEL}^{-/-}$line $(13 \% \pm 2.6 \%)$ compared with the $\mathrm{MEL}^{+/+}$line $(4.3 \% \pm 1 \%)$ (Supplemental Figure $5 \mathrm{~B})$. These results suggest that while complete absence of GATA6 may enhance cell proliferation, it also increases apoptosis, preventing further lung maturation. However, temporary knockdown of GATA6 does not increase the apoptotic marker expression (Supplemental Figure 5C). Reports using mouse models have shown that decreased GATA6 expression resulted in increased canonical Wnt signaling (15). In the $\mathrm{MEL1}^{-/-}$line, increased expression of the WNT target AXIN2 was observed in addition to BMP4, ID2, and FGF10 and the noncanonical Wnt receptor (FZD2) (Supplemental Figure $4 \mathrm{D})$, suggesting that other signaling pathways may also play a role. Thus, a complete loss of GATA6 leads to a dramatic effect on lung progenitor induction, indicating that GATA6 expression negatively affects NKX2.1 lung progenitor generation.

GATA 6 is required for differentiation into mature lung lineage cells. To define the role of GATA6 in the generation of mature lung lineages, GATA6-knockdown and -null cells were analyzed at day 26 of lung maturation (16) (Supplemental Figure 6A). The GATA6 siRNA-treated cells maintained increased NKX2.1 expression and increased the $\mathrm{SOX} 2{ }^{+} \mathrm{NKX} 2.1^{+}$proximal lung progenitors (Supplemental Figure 6, B and D). The GATA6-null line showed a dramatic decrease in NKX2.1 expression and cell number and displayed a very different morphology (Supple- 
A

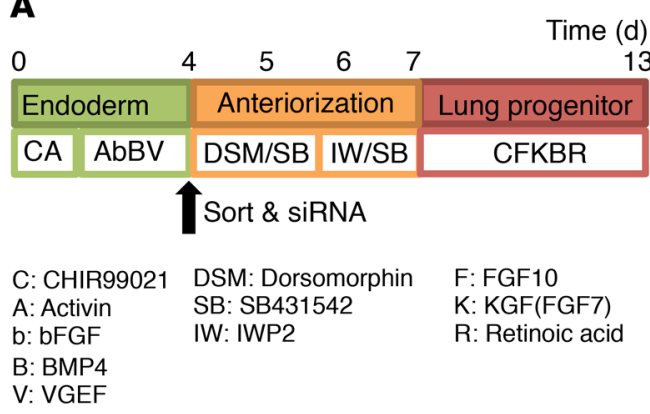

D

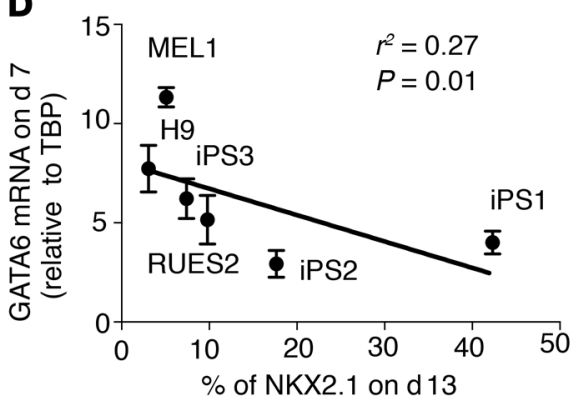

G

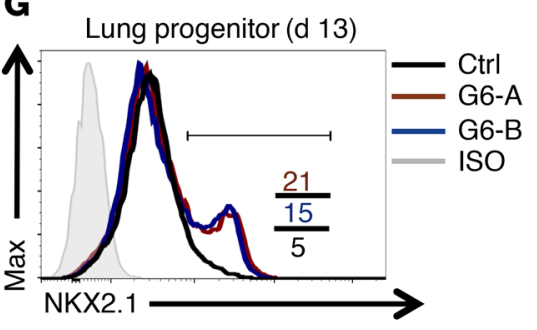

J Lung progenitor (d 13)
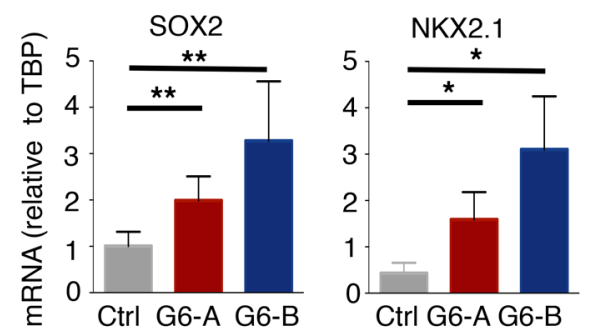

$\mathbf{E}$

H

$\mathbf{K}$
B
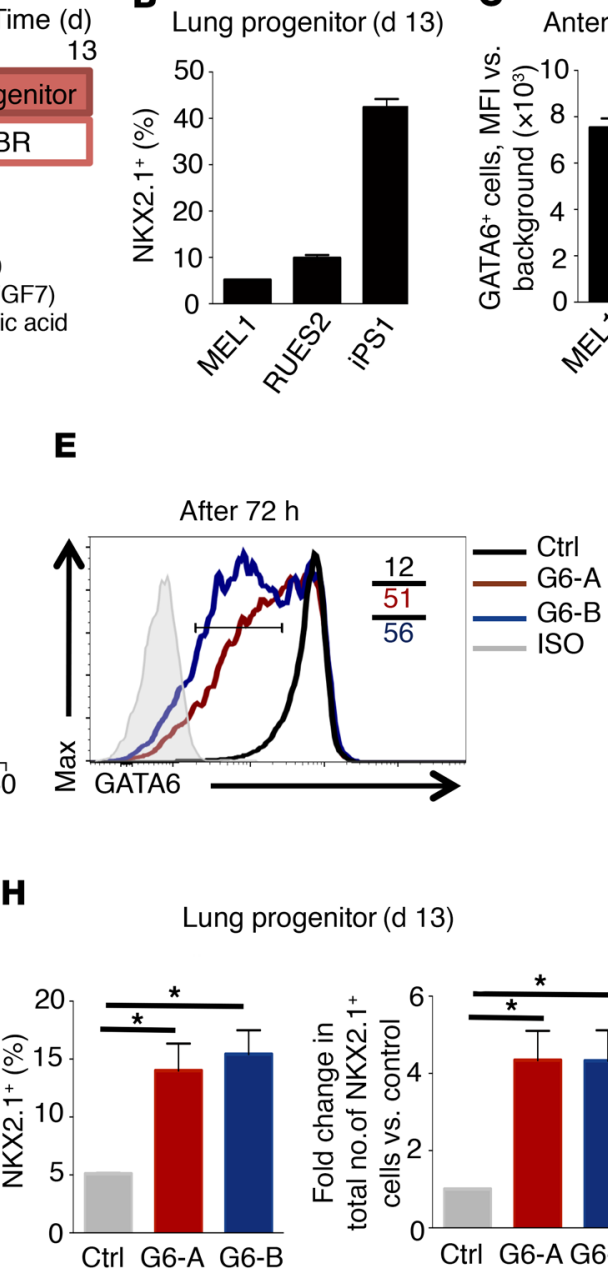

Lung progenitor (d 13)
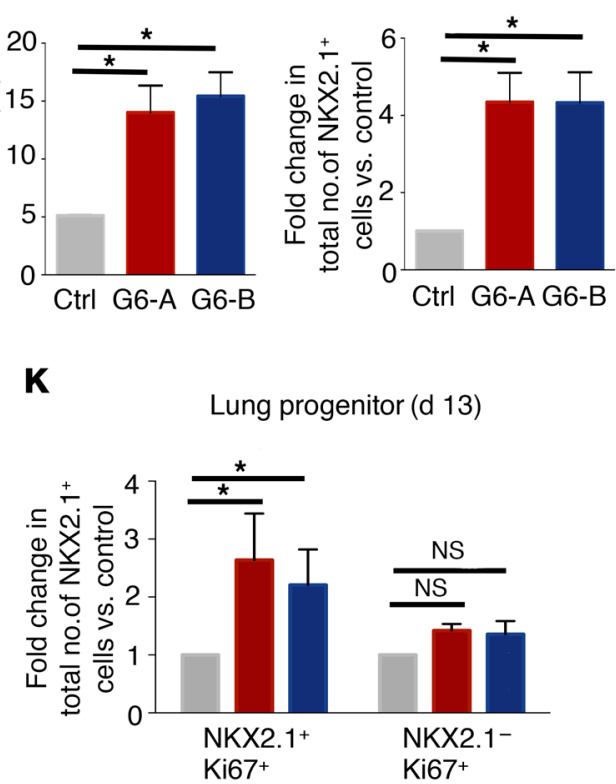

C Anteriorization (d 7)

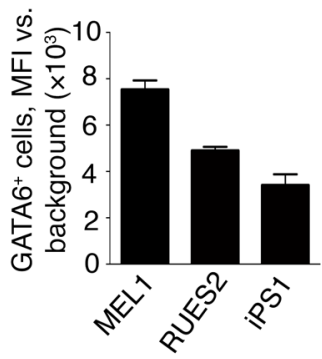

F

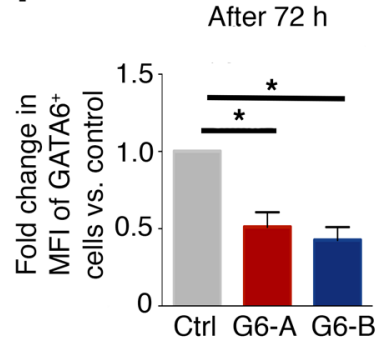

I

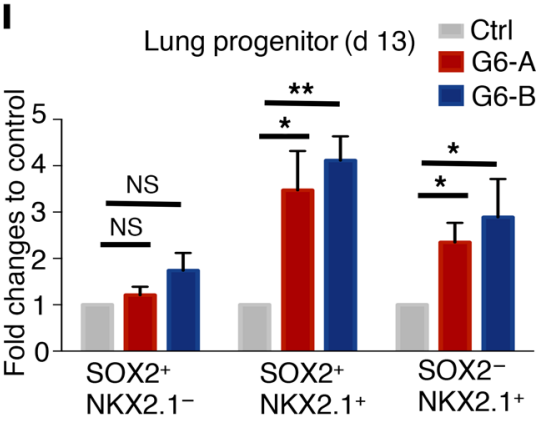

Figure 1. Low GATA6 expression during endoderm patterning enhances lung progenitor generation. (A) Schematic of the lung differentiation protocol. (B and C) The expression of NKX2.1 in lung progenitors (B) and GATA6 at anteriorization (C) from 3 human PSC lines $(n=7)$. (D) Linear regression analysis of GATA6 on day 7 versus NKX2.1 on day 13 from 6 WT human cell lines. (E and F) Transfection of 2 siRNAs against GATA6 (G6-A and G6-B) or control scrambled siRNA (Ctrl) at anteriorization stage in MEL1 ESCs. CATA6 expression (E) and quantification (F) after siRNA treatment $(n=4)$. (G) The expression of NKX2.1 in cells treated with GATA6 siRNAs. (H) Average expression and absolute cell number of NKX2.1+ cells $(n=5)$. (I) Coexpression of indicated populations treated with GATA6 siRNAs $(n=5)$. (J) SOX2 and NKX2.1 expression of cells treated with GATA6 siRNAs $(n=5)$. (K) Coexpression of NKX2.1 and Ki67 in cells treated with GATA6 siRNAs $(n=5)$. Data indicate the mean \pm SEM. ${ }^{*} P<0.05,{ }^{* *} P<0.01$ by 2 -tailed $t$ test.

mental Figure 6, B-E), which is not surprising considering the increased markers of apoptosis in the GATA6-null lines at the lung induction stage (Supplemental Figure 5, A and B). We also examined thyroid marker expression and did not observe the impact of GATA6 siRNA in driving thyroid fate at this stage (Supplemental Figure 7).
In order to examine whether GATA6 knockdown has a longterm impact for later lung maturation, we adopted a 3D culture (16) in $\mathrm{MEL}^{+/+}$cells (Supplemental Figure 8A). We observed a significant increase of NKX2.1 (>10-fold) and distal lung epithelium markers (surfactant protein C [SPC] $>100$-fold, surfactant protein B [SPB] >15-fold) (Supplemental Figure 8B). NKX2.1 expression 
A Definitive endoderm (d 4) MEL1+/+

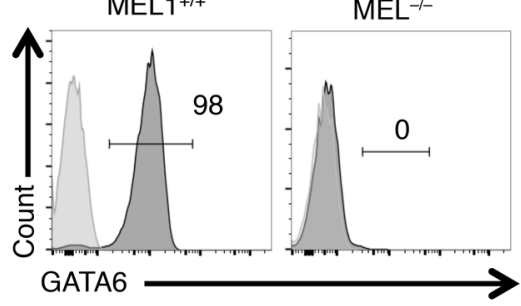

B

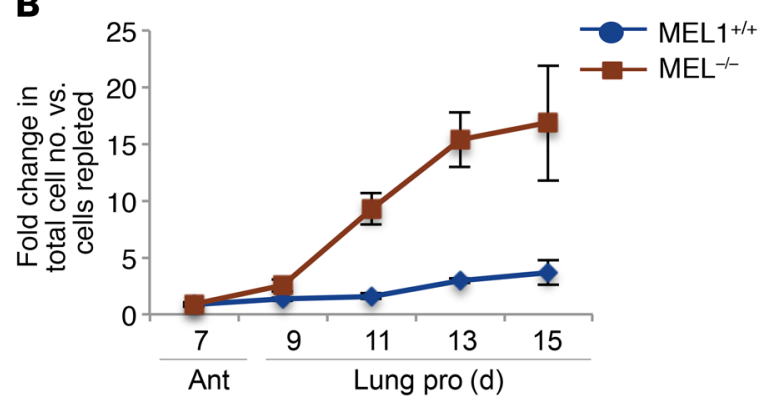

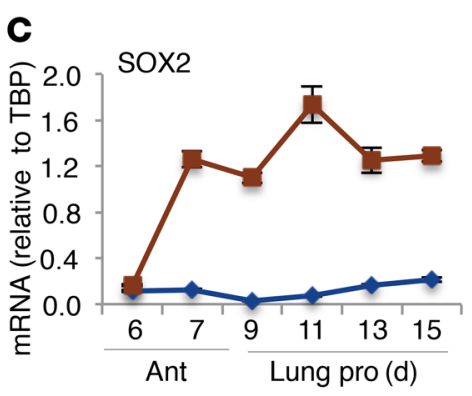

D

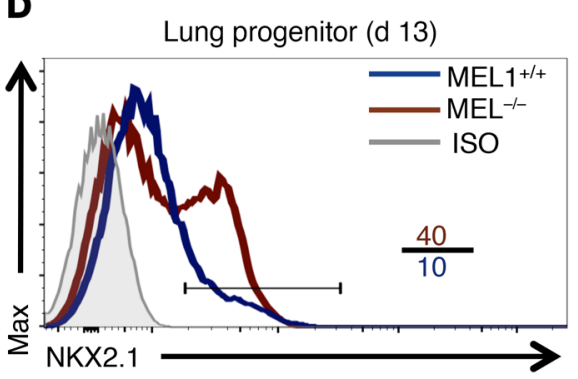

$\mathbf{F}$

Lung progenitor (d 9) (gated on total population)

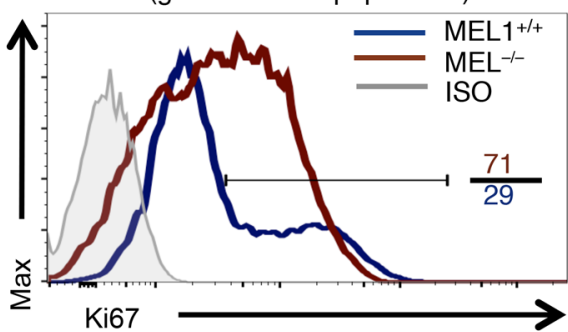

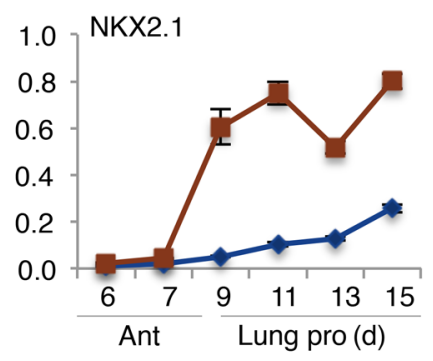
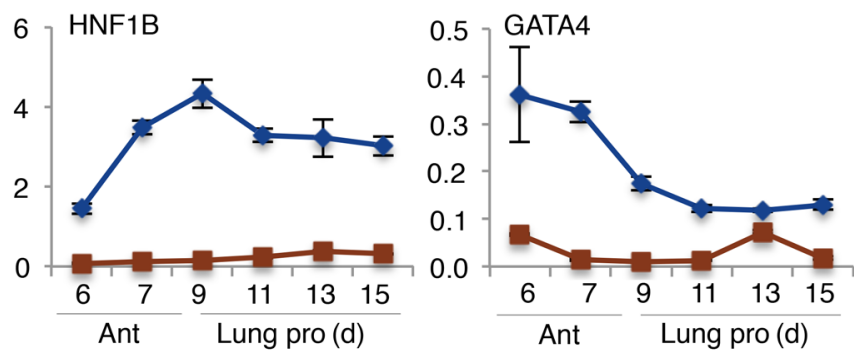

E
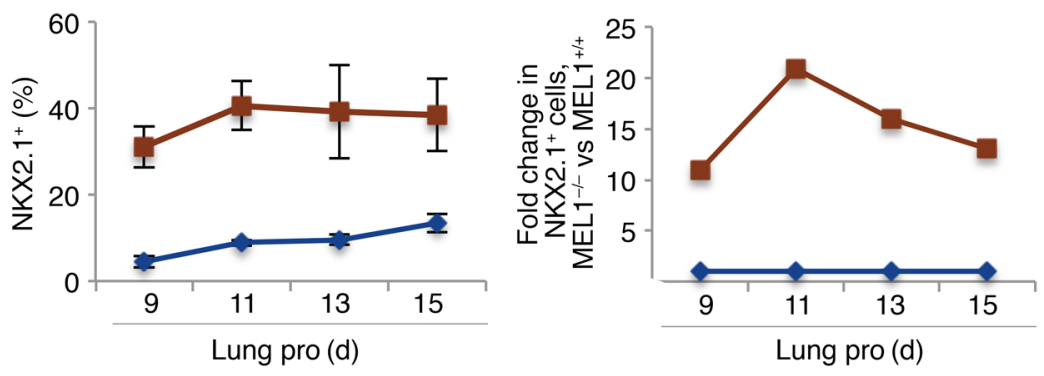

G
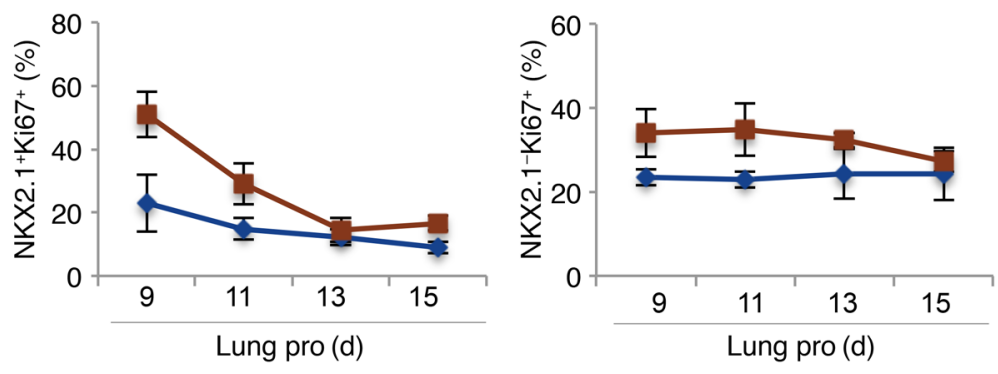

Figure 2. Complete loss of GATA6 during endoderm patterning enhances lung progenitor generation. (A) The expression of CATA6 on definitive endoderm in the $\mathrm{MEL}^{+/+}$and MEL1 ${ }^{-/-}$lines $(n=3)$. (B) Total cell number examined from both MEL1 ${ }^{+/+}$and MEL1 $1^{-/-}$lines $(n=6)$. (C) The expression of indicated markers in human $\mathrm{MEL}^{+/+}$and $\mathrm{MEL1}^{-/-}$lines during lung differentiation. Representative data are from 4 experiments. Expression of NKX2.1 examined on

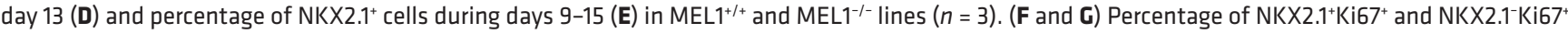

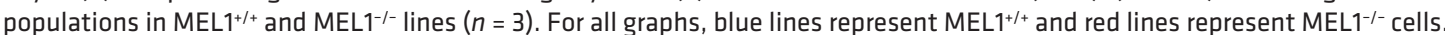

levels were very close to those seen in fetal lung, whereas SPB levels were approximately a third and SPC levels were still less than $1 \%$ of those found in vivo (Supplemental Figure 8C). Although low, SPC levels were comparable with those in current in vitro protocols of lung differentiation (17). When we examined pro-SPC by immunohistochemistry, we observed an overall increase of pro-SPC expression in the siRNA-treated cultures (Supplemental Figure 8D). Furthermore, siRNA treatment did not seem to affect either thyroid or neural markers (Supplemental Figure 8E). These data suggest that the NKX2.1+ population generated in the context of temporary GATA6 loss is competent for further lung maturation but that GATA6 has a role later during differentiation to allow lung maturation, and this idea is supported by mouse studies $(8,10)$.

Liver differentiation induced by GATA6 reexpression during anteriorization. To more closely examine how the timing of GATA6 expression impacts lung lineage commitment, GATA6 doxycycline-inducible (DOX-inducible) cells were generated in the MEL1 ${ }^{-/}$background (MEL1 ${ }^{-/-}$G6Tg) (Supplemental Figure 9A). GATA6 was expressed in about $30 \%$ of the day- 13 population, showing some transgene silencing but with protein levels compa- 
A

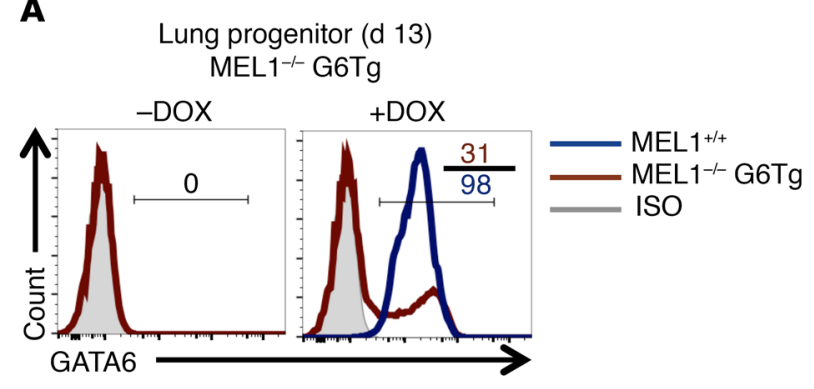

C

MEL1-/- G6Tg

(gated on GATA6 ${ }^{+}$)

(d 4) +DOX
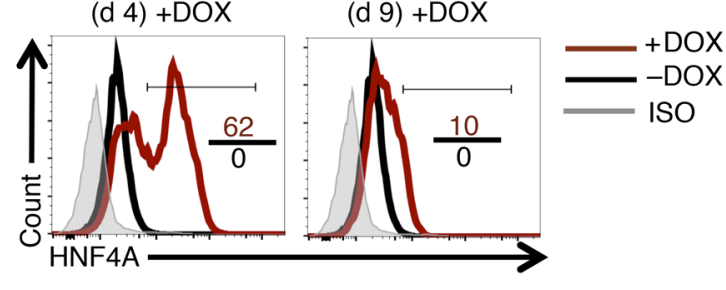

B
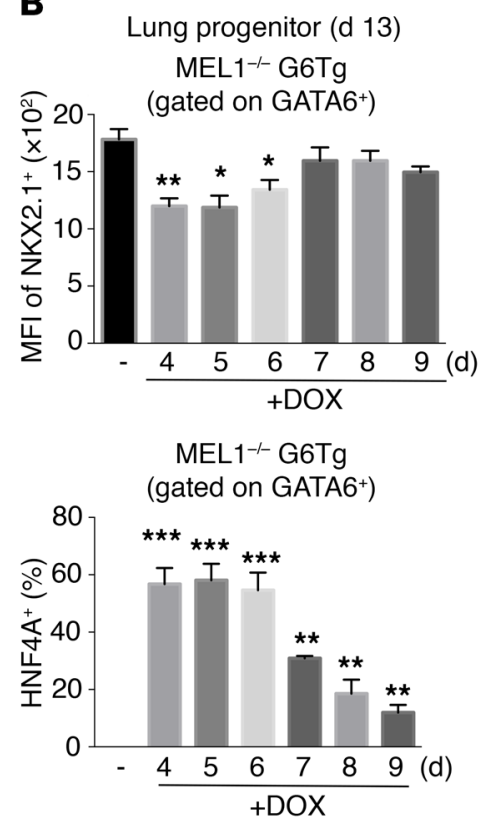

\section{D}

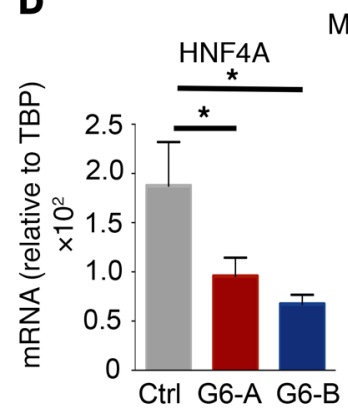

$\mathrm{MEL}^{+1++}$

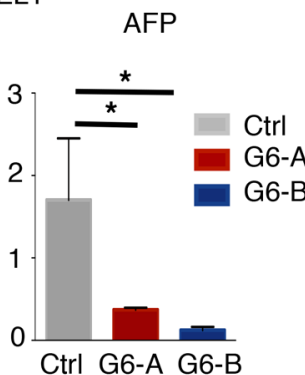

E

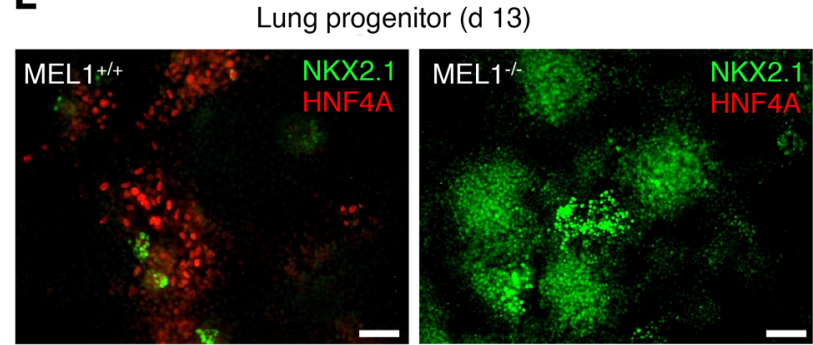

F
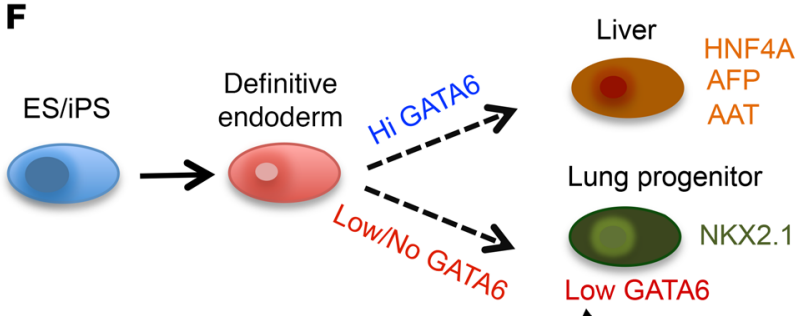

Lung progenitor
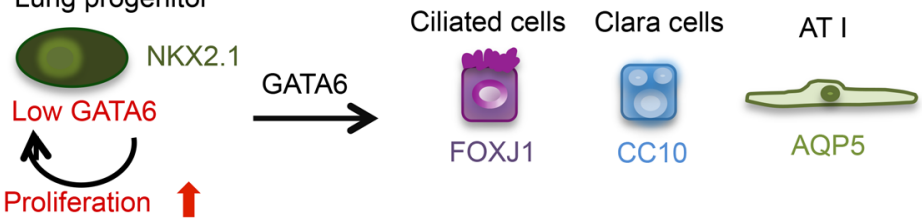

AT II

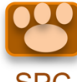

SPC

Figure 3. Reexpression of GATA6 during anteriorization induces liver development. (A) GATA6 expression in lung progenitors with and without doxycycline $(\mathrm{DOX})(n=3)$. (B) Expression of NKX2.1 within the GATA6 ${ }^{+}$population in cells treated with DOX starting from day 4 or day 9 onward and the MFI of NKX2.1 ${ }^{+}$ within the GATA6 ${ }^{+}$population $(n=3)$. (C) HNF4A expression in cells treated with DOX beginning at day 4 or day 9 and average expression of HNF4A in GATA6 ${ }^{+}$cells from DOX-treated cultures $(n=4)$. (D) Expression of hepatocyte markers HNF4A and AFP in human MEL1+/+ cells treated with GATA6 siRNAs $(n=5)$. Data indicate mean \pm SEM. ${ }^{*} P<0.05,{ }^{* *} P<0.01,{ }^{* *} P<0.005$ by 2 -tailed $t$ test. (E) Immunostaining of NKX2.1 and HNF4A of cells on lung progenitors from MEL1 ${ }^{+/+}$and MEL1 ${ }^{-/-}$lines $(n=4)$. Magnification: $\times 100$. Scale bars: $100 \mu \mathrm{m}$. (F) Model depicting impact of GATA6 on cell-fate decisions toward either hepatocyte or lung lineages.

rable to endogenous GATA6 (Figure 3A). Gating on these GATA6 ${ }^{+}$ cells, NKX2.1 expression was reduced only when DOX was begun at earlier time points (days 4-6) (Figure 3B) and the hepatocyte marker HNF4A was induced (Figure 3C). Increases in other liver markers such as AFP, GATA4, and HNF1B were also seen (Supplemental Figure 9B), suggesting that early induction of GATA6 drove cell fate toward the liver.
To confirm these findings and determine if other GATA family members can have a similar impact on lung specification, we reexpressed GATA6 or GATA4 in MEL1 ${ }^{-/-}$cells and found that expression of either family member led to decreased NKX2.1 expression and increased liver gene expression with a more profound effect when DOX was added earlier (Supplemental Figure 10, A-C). Confirmation of the role of GATA6 on endoderm patterning into 
the liver was also shown by significantly reduced expression of HNF4A and AFP in the siRNA-treated $\mathrm{MEL1}^{+/+}$line (Figure 3D). Lastly, examining 3 PSC lines demonstrated that the lines expressing the highest endogenous GATA6 levels during anteriorization have the highest levels of liver markers later in differentiation (Supplemental Figure 1D). To confirm whether GATA6 is involved in hepatocyte generation at the expense of lung differentiation, we costained NKX2.1 with HNF4A (Figure 3E and Supplemental Figure 1E). We observed few NKX2.1 $1^{+}$cells and mostly $\mathrm{HNF}_{4} \mathrm{~A}^{+}$ cells from the $\mathrm{MEL1}^{+/+}$line, no $\mathrm{HNF}_{4} \mathrm{~A}^{+}$expression in the $\mathrm{MEL1}^{-/-}$ line, and no coexpression. These results suggest that high GATA6 expression levels may induce hepatocyte generation at the expense of lung progenitor differentiation.

Our results suggest that GATA6 plays a critical role during human endoderm patterning (Figure 3F). A high level of GATA6 during anteriorization skews the cell fate toward the liver lineage, whereas a low level of GATA6 during anteriorization promotes lung lineage commitment with elevated SOX2 expression and expansion of lung progenitors. Supporting these results, a previous publication describing the generation of a SOX2 reporter hESC line found that lung progenitors were enriched in the SOX $2^{\mathrm{hi}}$ GATA6 ${ }^{\text {low }}$ population (18). Finally, GATA6 is necessary later in development for lung specification and maturation.

In the present study, we discovered that loss of GATA6 leads to expansion of a lung progenitor population derived from human PSCs. This phenomenon appears similar to one brought about by G1ME cells (19), where the lack of GATA1 enhances megakaryocyteerythroid progenitor expansion. While we demonstrate a 15 -fold expansion, it will be interesting to see if this novel lung progenitor population stemming from GATA6 $^{-/-}$cells may be capable of more extensive self-renewal with appropriate culture conditions that prevent the apoptosis seen in our cultures. Considering the requirement for GATA6 in the expression of lung terminal differentiation markers, GATA6 may be playing a role in the lung similar to the one GATA1 plays in the hematopoietic system (19), driving differentiation such that its absence allows expansion of progenitor populations. Nevertheless, transient knockdown of GATA6 during anteriorization seems to have a global positive effect in promoting lung lineage commitment by reducing hepatocyte lineage commitment in lines that have high endogenous GATA6. In summary, correct spatial-temporal GATA6 expression is critical during human lung development.

One of the problems with the current protocols for generating terminally differentiated lung cell types is that efficiency is highly variable from cell line to cell line. Our studies suggest that endog- enous GATA6 levels are critical for endoderm patterning and the ability for a given cell line to generate lung progenitors. By examining GATA6 expression during early endoderm patterning, it is fairly straightforward to determine which lines will generate lung progenitors with efficiency, allowing a simple method to pick the most robust lines (Figure 1D). Additionally, temporary GATA6 suppression during anteriorization greatly increases the efficiency of generating lung progenitors and reduces line-to-line variability. Combining transient GATA6 knockdown with the recently described cell-surface antigens $\left(C D 47^{+} \mathrm{CD} 26^{-}\right)(16)$ that are specific for the NKX2.1 $1^{+}$population will allow the robust generation of pure lung progenitors from virtually any human PSC line. These advances will aid the use of patient-derived iPSC lines with lung disorders, as it is currently difficult to efficiently generate lung progenitors from certain genetic backgrounds.

\section{Methods}

Details about experimental procedures are provided in the Supplemental Methods.

Study approval. The IRB of the Children's Hospital of Philadelphia (CHOP) approved the original generation of the human iPSCs with documented informed consent obtained from participants. Human control lung tissues without identifying information were obtained from the pathology core facility at CHOP.

\section{Author contributions}

CML designed research studies, conducted experiments, acquired data, analyzed data, and wrote the manuscript. SM, AT, JAM, and FLCD conducted experiments. DLF and PG supervised and designed research studies, analyzed data, and revised the manuscript.

\section{Acknowledgments}

We thank Chintan Jobaliya and Rebecca Meyer for cell sorting and Alyssa Gagne and Catherine Osorio Quintero for technical support at the human iPS cell core facility at CHOP. We thank the pathology core facility at CHOP for immunohistochemistry. This work was supported by the National Institutes of Health (grant U01HL110942 to PG) and a Ruth L. Kirschstein NRSA Individual Postdoctoral Fellowship (F32 HL120608 to CML).

Address correspondence to: Paul Gadue, Department of Pathology and Laboratory Medicine, Children's Hospital of Philadelphia, 3501 Civic Center Boulevard, Philadelphia, Pennsylvania 19104, USA. Phone: 267.426.9607; Email: gaduep@email. chop.edu.
1. Rackley CR, Stripp BR. Building and maintaining the epithelium of the lung. JClin Invest. 2012;122(8):2724-2730.

2. Minoo P, Su G, Drum H, Bringas P, Kimura S. Defects in tracheoesophageal and lung morphogenesis in Nkx2.1(-/-) mouse embryos. Dev Biol. 1999;209(1):60-71.

3. Rock JR, Hogan BL. Epithelial progenitor cells in lung development, maintenance, repair, and disease. Annu Rev Cell Dev Biol. 2011;27:493-512.

4. Yamamoto M, Ko LJ, Leonard MW, Beug H, Orkin $\mathrm{SH}$, Engel JD. Activity and tissue-specific expres- sion of the transcription factor NF-E1 multigene family. Genes Dev. 1990;4(10):1650-1662.

5. Arceci RJ, King AA, Simon MC, Orkin SH, Wilson DB. Mouse GATA-4: a retinoic acid-inducible GATA-binding transcription factor expressed in endodermally derived tissues and heart. Mol Cell Biol. 1993;13(4):2235-2246.

6. Shaw-White JR, Bruno MD, Whitsett JA. GATA-6 activates transcription of thyroid transcription factor-1. J Biol Chem. 1999;274(5):2658-2664.

7. Morrisey EE, et al. GATA6 regulates HNF4 and is required for differentiation of visceral endoderm in the mouse embryo. Genes Dev. 1998;12(22):3579-3590.

8. Keijzer R, et al. The transcription factor GATA6 is essential for branching morphogenesis and epithelial cell differentiation during fetal pulmonary development. Development. 2001;128(4):503-511.

9. Liu C, Morrisey EE, Whitsett JA. GATA-6 is required for maturation of the lung in late gestation. Am J Physiol Lung Cell Mol Physiol. 2002;283(2):L468-L475.

10. Koutsourakis M, Keijzer R, Visser P, Post M, Tibboel D, Grosveld F. Branching and differentiation defects 
in pulmonary epithelium with elevated Gata6 expression. Mech Dev. 2001;105(1-2):105-114.

11. Huang SX, et al. Efficient generation of lung and airway epithelial cells from human pluripotent stem cells. Nat Biotechnol. 2014;32(1):84-91.

12. Tiyaboonchai A, et al. GATA6 plays an important role in the induction of human definitive endoderm, development of the pancreas, and functionality of pancreatic $\beta$ cells. Stem Cell Reports. 2017;8(3):589-604.

13. Shi ZD, et al. Genome editing in hPSCs reveals GATA6 haploinsufficiency and a genetic interac- tion with GATA4 in human pancreatic development. Cell Stem Cell. 2017;20(5):675-688.e6.

14. Que J, Luo X, Schwartz RJ, Hogan BL. Multiple roles for Sox 2 in the developing and adult mouse trachea. Development. 2009;136(11):1899-1907.

15. Zhang Y, et al. A Gata6-Wnt pathway required for epithelial stem cell development and airway regeneration. Nat Genet. 2008;40(7):862-870.

16. Hawkins F, et al. Prospective isolation of NKX21-expressing human lung progenitors derived from pluripotent stem cells. JClin Invest. 2017;127(6):2277-2294.
17. McCauley KB, Hawkins F, Serra M, Thomas DC, Jacob A, Kotton DN. Efficient derivation of functional human airway epithelium from pluripotent stem cells via temporal regulation of Wnt signaling. Cell Stem Cell. 2017;20(6):844-857.e6.

18. Brafman DA, et al. Analysis of SOX2-expressing cell populations derived from human pluripoten stem cells. Stem Cell Reports. 2013;1(5):464-478.

19. Noh JY, et al. Inducible Gata1 suppression expands megakaryocyte-erythroid progenitors from embryonic stem cells. J Clin Invest. 2015;125(6):2369-2374. 